\title{
O Procedimento Selective Reminding na Avaliação da Memória e Aprendizagem: Um Levantamento de Estudos Brasileiros
}

\author{
Suelen Bordignon ${ }^{1}$ \\ Murilo Ricardo Zibetti \\ Clarissa Marceli Trentini \\ Universidade Federal do Rio Grande do Sul
}

\begin{abstract}
RESUMO - Os pesquisadores têm à sua disposição uma variedade de métodos que auxiliam a compreensão dos processos de memória e aprendizagem. O presente artigo tem como objetivo revisitar o procedimento de recordação seletiva, apresentando suas características e variações, e realizar um levantamento de estudos brasileiros que fizeram uso do mesmo. São apresentadas as diferenças entre a recordação seletiva e o procedimento padrão em tarefas de aprendizagem por recordação livre. Constatouse que, no Brasil, além de diferentes tarefas, são utilizadas formas de aplicações distintas do procedimento. A utilização da recordação seletiva apresenta relevância no estudo da memória e da aprendizagem, em especial na busca pela diferenciação entre as dificuldades normais de memórias e os déficits patológicos dessa função cognitiva.
\end{abstract}

Palavras-chave: selective reminding, recordação seletiva, FCRT, aprendizagem, memória

\section{Assessing Memory and Learning Using the Selective Reminding Procedure: A Survey of Brazilian Studies}

\begin{abstract}
Researchers have a wide variety of methods that help to understand the processes of memory and learning. This paper aims to revisit the selective reminding procedure by presenting its characteristics and variations, and carry out a survey of Brazilian studies that made use of it. Differences are presented between the selective reminding procedure and the standard procedure in the use of free recall learning tests. The study showed that, in Brazil, the selective reminding procedure uses, not only different tasks, but also different application methods. The use of selective reminding is relevant to the study of memory and learning, especially to differentiate normal memory difficulties from pathological deficits in this cognitive function.
\end{abstract}

Keywords: selective reminding, FCRT, learning, memory

A compreensão do modo como os seres humanos organizam e manipulam o conhecimento configura-se, ainda hoje, em um desafio para as ciências. Áreas diversas como a neuropsicologia, neurologia, psiquiatria, informática, fonoaudiologia, psicologia, entre outras, têm buscado elucidar os processos de organização do conhecimento. Dentre os construtos abordados por essas áreas, destacase a memória, que diz respeito à capacidade de codificar, armazenar/consolidar e recordar/evocar informações (Tulving \& Craik, 2000).

A codificação é o processo de aquisição de informações, é a tradução dos estímulos externos em códigos individuais (Brown \& Craik, 2000). Nessa etapa, interações do hipocampo e das regiões neocorticais como estruturas da região límbica do cérebro estão envolvidas (Markowitsch, 1995; McCormick, Moscovitch, Protzner, Huber, \& McAndrews, 2010), conferindo ao conteúdo uma elaboração mais personalizada, que pode estar associada a registros emocionais. Uma vez codificadas, as informações são arquivadas através do processo denominado armazenamento (Izquierdo, 2011). Nessa fase, há um fortalecimento das representações, formando-se um arquivo mental que pode ser acessado em momento oportuno via lembrança. As principais

1 Endereço para correspondência: Rua Ramiro Barcelos, 2600, Sala 119, Santa Cecília, Porto Alegre, RS, Brasil. CEP: 90.035003.E-mail: bordignon.suelen@gmail.com áreas cerebrais envolvidas na consolidação das memórias declarativas são o lobo temporal, o hipocampo e as conexões com outras áreas do cérebro, tais como córtex entorrinal, neocórtex e amígdala (Quevedo, Martins, \& Izquierdo, 2006). Por fim, é através da recordação que os conteúdos codificados e armazenados são acessados e recuperados (Brown \& Craik, 2000). As áreas envolvidas na recordação de memórias declarativas são, em geral, as mesmas utilizadas em sua formação, sendo que o hipocampo e a amígdala, com o passar do tempo, dão lugar a outras regiões do córtex (Izquierdo, Myskiw, Benetti, \& Furini, 2013).

A complexidade dos sistemas cognitivos faz com que pesquisadores desenvolvam e utilizem uma variedade de métodos que auxiliem a aproximação com seu objeto de estudo. Especificamente no estudo da memória, podem-se citar tarefas que utilizam diferentes procedimentos, como o controle da evocação através de testes de reconhecimento com escolha livre ou forçada, a recordação livre e serial, a utilização de diferentes tempos de apresentação dos itens, o uso de pistas na codificação, na recordação, ou em ambas, o uso do selective reminding (recordação seletiva), entre outros (Tulving \& Craik, 2000). O objetivo deste artigo é apresentar o procedimento de "recordação seletiva", relacionando-o com os marcos teóricos no momento de sua criação, suas características, variações e uso atual, bem como realizar uma revisão de estudos brasileiros que se utilizaram de testes ou tarefas com esse procedimento. 
Parte dos procedimentos atualmente utilizados na avaliação da memória e da aprendizagem surgiu com o advento do cognitivismo. Foi a partir dos anos 60 que os achados de pesquisas em memória, linguagem e outros processos psicológicos básicos, apontaram inconsistências com a compreensão behaviorista (Bower, 2000). No estudo da aprendizagem e memória, um dos procedimentos estudados foi o de recordação livre. Diferentemente da recordação serial, na qual a lista estudada é evocada na mesma ordem apresentada, na recordação livre, os itens (palavras ou imagens) podem ser evocados na ordem de preferência do participante. As tarefas de recordação livre apresentam três achados relevantes e que não podem ser avaliados através da recordação serial: efeitos de recência e primazia (Murdock, 1962), organização subjetiva da lista (Tulving, 1962) e a constatação de que a capacidade de armazenamento é maior do que a de recuperação (Tulving \& Pearsltone, 1966).

Os efeitos de recência e primazia estão relacionados, respectivamente, a uma maior probabilidade dos primeiros e dos últimos itens da lista estudada serem recordados (Murdock,1962). Uma hipótese para esse fenômeno seria a presença de dois sistemas distintos de memória: um de longa duração e outro de curta duração (Atkinson \& Shiffrin, 1968; Glanzer \& Cunitz, 1966). Ou seja, a recordação dos primeiros itens (primazia) ocorreria à medida em que os itens apresentados passassem para a memória de longa duração. Já os últimos itens seriam recordados por ainda estarem na memória de curta duração. A primazia sofreria maior influência de capacidade de armazenamento de longa duração e a recordação dos últimos dependeria de diversas variáveis como tempo entre a apresentação e a recordação e possíveis interferências que concorressem com a atenção na tarefa (Glanzer \& Cunitz, 1966).

Essa tendência ou facilitação da recordação das primeiras e últimas palavras da lista em detrimento das palavras centrais, gera uma curva denominada "curva de posição serial". Experimentos têm demonstrado que adultos normais apresentam essa curva quase que em forma de "U", enquanto idosos sadios tendem a apresentar uma curva próxima à forma de "J", demonstrando melhor recordação das últimas palavras da lista e, comparativamente aos adultos jovens, um rebaixamento da memória de longa duração (maiores dificuldades na recordação das primeiras palavras da lista) (Abrisqueta-Gomes, 2013). Na presença de patologias, essa curva pode se mostrar alterada, por exemplo, na demência da Doença de Alzheimer (DA). Em estudo realizado por Bueno, Bertolucci, Oliveira e Abrisqueta-Gomes (2008), foi demonstrado que, nos estágios iniciais da DA, é possível verificar um rebaixamento da primazia, em comparação com idosos sem a doença. Em estágios mais avançados da doença, observa-se também o comprometimento de recência.

Outro achado importante em tarefas de recordação é a organização subjetiva da lista (Tulving, 1962), que é atribuída ao processo cognitivo de organização dos itens para facilitar a memorização. A repetição da lista está relacionada a uma melhor aprendizagem em função da possibilidade de organizar as informações mentalmente, de modo que não aumenta a capacidade da memória, mas, ao unir alguns dos itens (transformando aglomerados), propiciaria a recordação de uma quantidade maior de itens
(Tulving, 1962). Essa organização é também conhecida como chunking e é dependente do conhecimento prévio de cada sujeito, que determina a forma como o conteúdo aprendido será manipulado (Miller, 1956). Além dessas considerações, estudos realizados com o procedimento de recordação livre demonstraram que a capacidade de armazenamento de itens em tarefas de memória é maior do que a capacidade de recordá-los. Através do uso de pistas é possível resgatar do armazenamento itens que não foram recordados de forma livre (Tulving \& Pearsltone, 1966).

A partir dessas descobertas, diferentes procedimentos foram sendo agregados às tarefas de recordação livre para uma melhor compreensão dos processos de memória e aprendizagem. Em especial, este artigo focará no procedimento de recordação seletiva.

\section{A Criação do Procedimento de Recordação Seletiva}

Em 1973, Buschke apresentou um procedimento de avaliação dos processos de memória e de aprendizagem, denominado recordação seletiva. Através do mesmo, pretendia compreender melhor os processos de armazenamento de longa duração, bem como de recuperação de longa e curta duração. Tais conceitos estão alicerçados nos estudos de Glanzer e Cunitz (1966) sobre dois sistemas de memórias distintos - um relacionado ao armazenamento de longa duração e outro ao de curta duração -, e de Tulving (1962) sobre a organização subjetiva do material, aplicados a tarefas de recordação livre.

O procedimento de recordação seletiva surgiu como crítica aos padrões até então adotados em tarefas de recordação livre para a compreensão da aprendizagem e da memória. Nestas, a lista a ser aprendida era reapresentada integralmente a cada novo ensaio, seguido de recordação livre. O procedimento de recordação seletiva tem como principal característica a reapresentação somente de itens não evocados em um ensaio imediatamente anterior. Como exemplo, ao realizar a tarefa de aprendizagem verbal, é apresentada uma lista de palavras e solicitada a recordação dos itens de forma livre. Os itens não evocados naquele ensaio são reapresentados. Nova recordação da lista e reapresentação de itens não evocados é realizada. Esse padrão é mantido até que se consiga a recordação de todos os itens ou até que se alcance o número de ensaios pré-estabelecido (Buschke, 1973; Buschke \& Fuld, 1974). Essa alteração proporcionada pelo procedimento de recordação seletiva propicia redução do tempo de aplicação, apresentação personalizada de acordo com o desempenho ao longo do teste e principalmente uma maior rapidez na aprendizagem da lista (MacLeod, 1985). Essas vantagens se devem ao direcionamento da atenção para estímulos ainda não aprendidos.

A principal crítica ao procedimento tradicional era que esse não conseguia distinguir entre a recuperação do armazenamento de curta duração e a do armazenamento de longa duração. Ao desenvolver o procedimento de recordação seletiva, Buschke (1973) teorizou que os itens que eram recuperados sem necessitarem de nova apresentação pertenciam a um armazenamento de longa duração. Através da reapresentação somente dos itens não recordados, 
aumentaria a probabilidade de aprendizagem dos mesmos, visto que a atenção estaria direcionada para os itens não recuperados (Buschke \& Fuld, 1974).

Destaca-se que, na literatura da época, já existiam críticas ao procedimento padrão e estudos que se utilizavam de tarefas semelhantes à recordação seletiva. Uma técnica denominada "dropout" (Battig, 1965) estava presente em estudos com tarefas de pares associados. Tanto no procedimento de recordação seletiva como no "dropout", os itens corretamente recordados em um ensaio são removidos no ensaio posterior (MacLeod, 1985). Os estudos que se utilizam das técnicas de "dropout" ainda continuam referenciados na literatura de pares associados, especialmente em pesquisa básica (Karpicke \& Roediger, 2008; Pyc \& Rawson, 2011), enquanto que os estudos com recordação seletiva estão mais presentes na pesquisa aplicada (Di Stefano et al., 2014; Lemos, Duro, Simões, \& Santana, 2014), em tarefas visuais com uso de pistas ou mesmo de recordação de listas de palavras.

O procedimento de recordação seletiva apresenta algumas vantagens em relação aos procedimentos padrão, em especial na avaliação de pessoas idosas ou com algum comprometimento em funções cognitivas. Em especial, busca diferenciar processos normais de memória dos patológicos devido a condições clínicas (p. ex. Demência da DA, Declínio Cognitivo Leve, Acidente Vascular Cerebral). Nesse sentido, o procedimento busca controle da aprendizagem, através do direcionamento da atenção ao material na fase de estudo e também pela reapresentação de itens não recordados. A reapresentação somente de itens não recordados evita fadiga e desinteresse em razão da reaprendizagem e também reduz significativamente o tempo de testagem. Outro diferencial do procedimento diz respeito à avaliação da recuperação de curto, médio e longo prazo. Em tarefas com uso de pistas semânticas, busca-se a facilitação da codificação e auxílio posterior na recordação através de pistas para itens não recuperados. Por fim, a não reapresentação de itens já recordados auxilia a compreensão do conteúdo que foi aprendido pelo avaliando.

O procedimento de recordação seletiva foi primeiramente apresentado através de uma lista de 20 palavras de uma mesma categoria (animais de quatro patas). Os itens da lista eram lidos aos participantes com velocidade de apresentação de 2 segundos. Em seguida, era realizada a recordação livre dos itens e reapresentação daqueles não recordados. A lista de palavras, que tinha itens pertencentes a uma mesma categoria semântica, em seguida foi alterada, tendo em vista a possibilidade de acerto ao acaso, já que a categoria não suportaria muitos exemplares (Buschke, 1973). A Figura 1

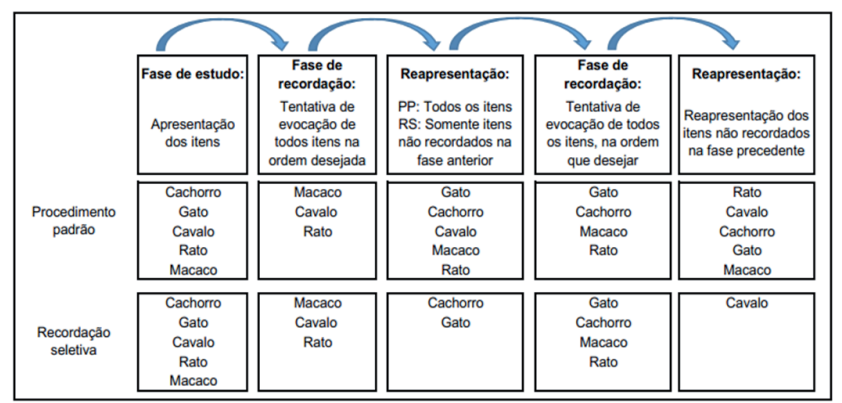

Figura 1. Comparação entre procedimento padrão e o procedimento de recordação seletiva inicialmente proposto apresenta de forma esquemática uma comparação entre o procedimento padrão e o de recordação seletiva inicialmente proposto. Somente a título de ilustração, foram utilizadas apenas cinco das 20 palavras da tarefa de Buschke (1973).

Através de uma tabela de pontuação, conseguiram demonstrar escores, tais como, somatório dos itens recuperados em cada ensaio, armazenamento de longa duração, recuperação de longa duração, recuperação de curta duração, aprendizagem da lista, recuperação de longa duração randômica, número de palavras reapresentadas em cada ensaio e intrusões. O somatório dos itens recordados diz respeito ao número de itens evocados em cada ensaio. A recordação de um item pela segunda vez consecutiva, sem reapresentação, demonstra que o item pertence ao armazenamento de longa duração, e sua evocação será denominada de recuperação de longa duração. A recordação de um item não evocado consecutivamente por duas vezes refere-se à recuperação de curta duração. A aprendizagem da lista diz respeito aos itens consistentemente recordados ao longo da tarefa sem a necessidade de nova apresentação e está relacionada à organização subjetiva da lista (Tulving, 1962). A recuperação de longa duração randômica está relacionada aos itens evocados do armazenamento de longa duração que não foram consistentemente recuperados. $\mathrm{O}$ número de palavras reapresentadas em cada ensaio demonstra o número de itens não recordados de forma livre. Por fim, as intrusões referem-se a itens que não estavam presentes na lista apresentada, mas foram referidos pelo participante (Buschke \& Fuld, 1974). Há uma ascensão dos itens evocados consistentemente e uma redução da recuperação de longa duração randômica ao longo dos ensaios em participantes normais, representando aprendizagem na tarefa (Buschke, 1973).

Ainda hoje, o procedimento de recordação seletiva vem sendo utilizado, seja da forma como foi proposto inicialmente, como também através de variações que foram sendo realizadas ao longo do tempo. Além disso, alguns instrumentos que fazem uso do procedimento, não levam em consideração todos os escores inicialmente preconizados.

\section{Características e Variações do Procedimento de Recordação Seletiva}

A utilização do procedimento é conhecida principalmente através dos nomes Selective Reminding Test (SRT), Buschke Selective Reminding Test ou Verbal Selective Reminding Test (VSRT). Apesar de ser nomeado como teste, trata-se de um procedimento, ou seja, uma maneira de avaliar a memória e a aprendizagem. As listas de palavras estão disponíveis para que os pesquisadores possam produzir seu próprio material (Strauss, Sherman, \& Spreen, 2006). Tem como objetivo principal a avaliação da aprendizagem verbal e da memória utilizando listas de palavras através de múltiplos ensaios (Lezak, 1995). Além das diferentes nomeações que recebe, novas formas de aplicação foram desenvolvidas ao longo do tempo, como, por exemplo, com modificações no número de ensaios e itens, bem como na natureza dos estímulos (Tabela 1). 
Atualmente, a versão mais utilizada é a apresentação da tarefa através de uma lista com 12 palavras não relacionadas. Um dos procedimentos de aplicação bastante utilizado é o de Hannay e Levin (1985), na qual a apresentação é feita durante 12 ensaios ou até que o avaliando consiga recordar toda a lista de 12 palavras por três ensaios consecutivos. Em seguida, é realizada a recordação com pistas através de cartões contendo as duas ou três primeiras letras de cada palavra da lista. Após é administrada uma tarefa de reconhecimento de múltipla escolha, contendo palavras da lista, palavras homônimas, sinônimas e distratoras. Por fim, é solicitada a recordação tardia, após 30 minutos.

Tendo em vista que o número de ensaios na realização da tarefa acarreta fadiga aos avaliandos e necessidade de maior tempo para aplicação, estudos foram realizados para avaliar a possibilidade de redução do número de ensaios de 12 para seis. Os escores obtidos em ambas as formas são igualmente representativos, excetuando-se o escore de recordação randômica, o qual é mais dependente da quantidade de ensaios. O procedimento com seis ensaios tem sido mais utilizado (Larrabbe et al., 2000; Morales et al., 2010).

Normas norte-americanas foram criadas para esses procedimentos, sendo que estão disponíveis normas para adultos e idosos com idades entre 18 e 91 anos (Larrabee et al., 2000). Versões adaptadas para crianças foram construídas com redução do número de palavras e de ensaios. (Crodfelter et al., 1987; Morgan, 1982). Além disso, normas para diferentes populações, como a espanhola (Campo \& Morales, 2004) e a hebraica foram desenvolvidas (Gigi et al., 1999).

Algumas características sociodemográficas estão relacionadas ao desempenho no Selective Reminding Test. Com o aumento da idade, há um declínio nos escores de recordação da lista, apesar do reconhecimento ser menos afetado (Campo \& Morales, 2004). Em relação ao sexo, mulheres apresentam melhor desempenho do que homens (Campo \& Morales, 2004). O quociente de inteligência apresenta correlações moderadas com os escores das tarefas (Bishop, 1990). No que tange à escolaridade, os dados não são conclusivos (Lezak, 1995).

Tabela 1. Diferentes apresentações do procedimento de recordação seletiva

\begin{tabular}{|c|c|c|c|c|c|}
\hline autores & $\mathbf{n}^{0}$ itens & natureza & $\begin{array}{c}\text { prancha } \\
\text { (categorias) }\end{array}$ & $n^{0}$ ensaios & características/escores \\
\hline Buschke (1973) & 20 & palavras & 1 (única) & não mencionado & $\begin{array}{l}\text { Duas variações: (a) selective } \\
\text { remining e (b) restrictive } \\
\text { reminding } \\
\text { Escores: Armazen. LD, } \\
\text { Recuperação (por ensaio, CD, } \\
\text { LD e randômica), aprendizagem } \\
\text { da lista }\end{array}$ \\
\hline Buschke e Fuld (1974) & 10 & palavras & 1 (única) & não mencionado & $\begin{array}{l}\text { Escores: (a) selective } \\
\text { reminding: idem a Buschke } \\
(1973)+\text { número de } \\
\text { reapresentações } \\
\text { (b) restrictive reminding: } \\
\text { Armazen. LD, Recuperação } \\
\text { (LD randômica, por ensaio, } \\
\text { com e sem apresentação), } \\
\text { aprendizagem da lista) }\end{array}$ \\
\hline Buschke (1984) & 12 & desenhos & 1 (diferentes) & $\begin{array}{l}6 \text { (mas refere uso } \\
\text { livre do número de } \\
\text { ensaios) }\end{array}$ & $\begin{array}{l}\text { Ensaios livre e com pistas } \\
\text { Controle de variáveis. Record. } \\
\text { livre e total (com pistas) }\end{array}$ \\
\hline Hannay e Levin (1985) & 12 & palavras & 1 (diferentes) & $\begin{array}{l}12 \text { (ou } 3 \text { acertos } \\
\text { consecutivos) }\end{array}$ & $\begin{array}{l}\text { Record. com pistas, tarefa de } \\
\text { reconhecimento de múltipla } \\
\text { escolha e record. tardia }\left(30^{\prime}\right)\end{array}$ \\
\hline Grober e Buschke (1987) & 16 & desenhos & 4 (diferentes) & 3 & $\begin{array}{l}\text { Ensaios livre e com pistas } \\
\text { nome do item junto ao desenho } \\
\text { Record. Imediata com pistas, } \\
\text { record. livre, total, consistente e } \\
\text { reconhecimento }\end{array}$ \\
\hline Petersen et al. (1992) & 16 & desenhos & 1 (diferentes) & 6 & $\begin{array}{l}\text { Ensaio livre e com } \\
\text { pistasRecord. livre e com pistas } \\
\text { a cada ensaio e tardia em } 30 \text { ', } \\
\text { recuperação LD, consistente, }\end{array}$ \\
\hline
\end{tabular}


Destaca-se a utilização do procedimento com diversos objetivos de avaliação: em mudanças cognitivas no teste de novas drogas (Culang-Reinlieb, Sneed, Keilp, \& Roose, 2012; Krupp et al., 2011), disfunções de memória em pacientes com doenças neurológicas (Bell, Fine, Seidenberg, \& Hermann, 2005; Solanto et al., 2007), na avaliação de práticas preventivas através de estímulos cognitivos, com o intuito de atrasar os declínios cognitivos em estágios pré-clínicos de demência (Pillai et al., 2011). Também tem sido utilizado para avaliar a diferenças no funcionamento neurocognitivo em patologias ou subtipos (Almeida et al., 2007) e no auxílio ao diagnóstico de doenças neurodegenerativas (Deloire et al., 2006; Wenger, Negash, Petersen, \& Petersen, 2010). Nos estudos de avaliação de intervenções, nos quais as tarefas foram reaplicadas em diferentes momentos, os autores se utilizaram de grupos controle ou grupo de comparação, buscando controlar o viés do efeito de prática (CulangReinlieb et al., 2012; Krupp et al., 2011; Pillai et al., 2011).

Além das variações do procedimento já apresentadas, também tem sido bastante empregado o uso de pistas categóricas para avaliar armazenamento e recordação. Em 1984, Buschke utilizou-se do procedimento de recordação seletiva no desenvolvimento de uma tarefa que buscava controlar as variáveis que interferem no processo de memória e aprendizagem, tais como atenção, processamento cognitivo e estratégias cognitivas. Essa manipulação sugeria uma confiabilidade maior aos resultados no que diz respeito aos construtos estudados.

Assim, Bushcke (1984) desenvolveu uma tarefa a partir de 12 itens visuais, cada um pertencente a uma categoria semântica distinta, as quais serviam de pistas para codificação. Inicialmente, solicitava aos participantes que localizassem em uma prancha e nomeassem cada um dos itens de acordo com a categoria apresentada. Ao realizar a busca, o participante estaria sendo induzido a olhar algumas vezes para cada item da prancha. Através da pista e da solicitação de nomeação, buscavam realizar uma codificação, controlando, assim, a forma com que essas informações estariam sendo processadas, induzindo também uma estratégia de codificação da informação a ser armazenada. Após 60 segundos de tarefas distratoras, uma evocação livre era solicitada e, para itens não recordados, a pista categórica era apresentada na tentativa de recuperar os itens armazenados. Seis ensaios eram realizados, através de recordações livres e com pistas, sendo que, entre os ensaios, tarefas distratoras eram realizadas. Para itens não recordados em cada ensaio, mesmo após apresentação da pista, a prancha era reapresentada e com o auxílio de pistas, nova codificação era realizada. Esse procedimento foi aplicado em pacientes com DA e em grupo sem a doença, porém o efeito de teto no escore do total recordado (livre e com pistas) nos grupos prejudicou a interpretação de alguns dados. De acordo com os resultados, o autor sugeriu que pacientes com DA apresentam habilidades para codificar e recordar informações quando as condições são controladas e estimuladas (através do foco de atenção, codificação mais profunda) e que as pistas podem auxiliar e ser úteis na avaliação da capacidade de memória e aprendizagem.

Três anos depois, Grober e Buschke (1987) apresentaram um novo formato para a tarefa, a qual se assemelha com o instrumento atual chamado Free and Cued Selective Reminding Test with Immediate Recall (FCSRT, Grober, Hall, Sanders, \& Lipton, 2008). Tendo em vista as limitações das interpretações dos resultados em estudos anteriores, o número de itens foi acrescido para 16. O formato atual do instrumento apresenta quatro cartões, cada qual com quatro figuras. Cada um dos itens está relacionado a uma categoria semântica distinta. $\mathrm{O}$ avaliador apresenta o primeiro cartão e menciona a categoria (p. ex. frutas), para qual o avaliando deve buscar o item relacionado (p. ex. uva) e nomeá-lo. Após a nomeação dos quatro itens desse primeiro cartão, o mesmo é retirado do campo de visão do avaliando e uma recordação através de pistas semânticas é realizada (nesse momento não há recordação livre), ou seja, o avaliador apresenta a categoria semântica e pede que o avaliando mencione o item associado. Para itens não recordados, nova busca através da categoria é realizada, com a reapresentação do cartão. Nova recordação com pista é realizada para itens não recordados e, caso o avaliando não consiga recordar novamente, o avaliador apresenta o par (categoria e item) e uma última tentativa é realizada. Em seguida, os demais cartões com quatro desenhos cada são apresentados, e segue-se o mesmo procedimento acima mencionado. Posteriormente, três ensaios de recordação são administrados, cada um com a apresentação de pistas para itens não recordados, configurando o uso da recordação seletiva. Caso o item não seja recordado, o avaliador apresentava o par ao participante. Por fim, uma tarefa de reconhecimento "sim-não" é realizada.

Atualmente, o FCSRT (Grober et al., 2008) é um teste que apresenta destaque internacional. Ele tem sido utilizado para identificar comprometimento cognitivo leve (Grober, Sanders, \& Lipton, 2010) e comprometimentos de memória em estágio pré-clínico da DA (Grober et al., 2008). O FCSRT é incluído no Compêndio de Neuropsicologia (Strauss, Sherman, \& Spreen, 2006) como uma variação do procedimento de recordação seletiva, porém Grober et al. (1997) fazem uma comparação e distinção entre o FCSRT e o procedimento recordação seletiva, afirmando que, através da fase inicial de codificação e uma recordação imediata através de pistas, há uma facilitação na recordação dos itens. Os participantes, idosos normais, recordaram duas vezes mais itens do armazenamento de longo prazo no FCSR do que na recordação seletiva, apontando possivelmente para uma maior sensibilidade como preditor à demência da DA anos mais tarde.

Uma pequena variação do procedimento foi realizada por Petersen, Smith, Kokmen, Ivnik e Tangalos (1992). A codificação dos itens era realizada através da apresentação de 16 itens visuais em uma mesma prancha. Cada um dos itens era codificado com uma pista categórica semântica e, em seguida, era solicitada a recordação livre dos itens e com pistas. Para aqueles não recordados, as pistas eram apresentadas juntamente com a prancha contendo os itens. Tal procedimento era realizado durante seis ensaios e uma evocação tardia era realizada após 30 minutos.

Internacionalmente existem duas baterias de avaliação que utilizam o método. O subteste Lista de Palavras da Learning and Memory Battery (Tombaugh \& Schmidt, 1992), utiliza-se de 15 palavras de diferentes categorias. Usa o procedimento de recordação seletiva com a utilização de 
pistas, através de cinco ensaios ou até que o sujeito recorde todas as palavras duas vezes consecutivas. O teste Memory Test for Older Adults - MTOA (Hubley \& Tombaugh, 2002), que é usado na avaliação de pessoas acima de 55 anos e apresenta uma subescala de lista de palavras em dois formatos, um longo e um curto. $\mathrm{O}$ formato longo faz uso de 15 palavras e cinco ensaios, enquanto que a aplicação curta se utiliza de 10 palavras e três ensaios. As palavras são lidas e, em seguida, solicita-se a recordação. Para as palavras não recordadas, a categoria a qual pertence o item é apresentada como pista. Existe uma recordação tardia após 10 minutos e uma tarefa de reconhecimento com o mesmo número de palavras alvo e distratores.

É importante destacar que o procedimento de recordação seletiva vem sendo bastante utilizado em tarefas e testes para diagnóstico diferencial entre declínio natural de memória decorrentes do envelhecimento normal e de déficits patológicos de memória, bem como na predição de demências. Estudos com o FCSRT têm demostrado seu potencial preditivo para DA, bem como para a diferenciação de demências da DA de outros subtipos (Grober et al, 2008b; Grober, 2010). Os escores de recordação livre fazem uma melhor discriminação entre demência e não demência e se apresentam como melhor preditor de demência de DA (Grober et al., 2010; Grober, Lipton, \& Crysta., 2000), enquanto que os escores de recordação total diferenciam melhor os pacientes com demência do tipo DA daqueles com demência vascular, demonstrando que ambos os quadros clínicos apresentam prejuízos em recordação livre, porém os pacientes com DA não conseguem se beneficiar da aprendizagem controlada propiciada pelo teste (Grober et al, 2008b).

Além do procedimento de recordação seletiva, Buschke e Fuld (1974) desenvolveram outro método de recordação livre, denominado "restricted reminding" (recordação restrita). Tinham como principal interesse avaliar déficits de memória e aprendizagem e identificar a fase do processo de aprendizagem que estaria comprometida: codificação, armazenamento ou recordação. A diferença, porém, entre os dois procedimentos é que, na recordação restrita, a reapresentação somente é realizada para aqueles itens que nunca foram recordados. Ou seja, a partir do momento que apresentar a primeira recordação, independentemente de ser ou não recordado nos ensaios seguintes, o item não será mais apresentado. Apesar de ser um procedimento que tem valor na compreensão de como os sujeitos armazenam e recordam listas de palavras, não tem sido muito utilizado pelos pesquisadores, talvez pelo fato de não propiciar a aprendizagem através da reapresentação dos itens.

A aplicação de ambos os procedimentos, recordação seletiva e recordação restrita, aos mesmos pacientes propiciou o entendimento de que itens não evocados em uma tentativa podem ser evocados em tentativas posteriores, mesmo sem a sua reapresentação. Esses resultados estão associados ao escore de recordação randômica de longo prazo. Tais achados sugerem que há um armazenamento de longa duração dessas informações e que as falhas estão relacionadas à recordação e não ao armazenamento (Buschke \& Fuld, 1974). Esse fenômeno de incremento ou melhora da performance de recordação ao longo de um teste é denominado "hipermnésia"
(Payne \& Roediger, 1987). Estudos na literatura de pesquisa básica são realizados através tarefas com múltiplos ensaios de recordação após um ensaio de aprendizagem. Diferenciase do procedimento de recordação restrita pela utilização de tarefas distratoras entre a apresentação dos estímulos e a primeira recordação e pelos intervalos de descanso entre as fases de recordação, nos quais os avaliandos são solicitados a repassarem mentalmente os estímulos da fase de aprendizagem (Remigio, 2010).

\section{O Procedimento de Recordação Seletiva e suas Variações no Brasil}

Embora o método de recordação seletiva tenha sido utilizado como parte integrante de tarefas e testes desenvolvidos internacionalmente, no Brasil, ainda é pouco utilizado e citado na literatura como tal. Listas de palavras com o procedimento clássico, de reapresentação de todas as palavras em cada ensaio, são mais conhecidos e estudados, como é o caso, por exemplo, do subteste Memória de Lista de Palavras da Bateria CERAD (Bertolucci, Okamoto, Neto, Ramos, \& Brucki, 1998; Ribeiro, Oliveira, Cupertino, Neri, \& Yassuda, 2010) e do Teste de Aprendizagem AuditivoVerbal de Rey (RAVLT) (Malloy-Diniz, Lasmar, Gazinelli, Fuentes, \& Salgado, 2007).

Em um levantamento realizado na base de dados Scielo e uma busca no site Google Scholar com os termos "Buschke", "selective reminding", "SRT", "BSRT", "FCSRT", "recordação seletiva" sem delimitador de ano de publicação, objetivou-se identificar estudos brasileiros que se utilizaram do procedimento de recordação seletiva. Alguns estudos nacionais encontrados foram conduzidos com o procedimento desenvolvido por Buschke em 1973 e Buschke e Fuld em 1974 para avaliação da memória episódica. Dessa forma, essas tarefas não fazem uso de pistas e se utilizam da reapresentação somente dos itens não recordados no ensaio imediatamente anterior. Por exemplo, Almeida et al. (2007) investigaram a relação da memória em níveis subclínicos de hipotireoidismo; Zachi, Ventura, Faria e Taub (2007) relacionaram prejuízos de memória em indivíduos com intoxicação por vapor mercúrio; Cunha, Nicastri, Gomes, Moino e Peluso (2004) identificaram déficit de memória em dependentes de cocaína e crack, Brook, Borela e Fragoso (2011) avaliaram o desempenho cognitivo em pacientes com Esclerose Múltipla e Netto et al. (2013) avaliaram a memória episódica em um treinamento de memória de trabalho (Tabela 2).

Outra parte dos estudos brasileiros que utilizou a recordação seletiva baseou-se nos procedimentos de Petersen et al. (1992), os quais fazem uso de pistas para codificação e há uma recordação com pistas para itens não recordados de forma livre. Em geral, os estudos que utilizaram esse procedimento relacionaram o desempenho cognitivo de idosos a variáveis sociodemográficas. Encontraram-se estudos de investigação longitudinal do desempenho de memória em indivíduos acima de 80 anos (Argimon \& Stein, 2005), investigação da relação entre memória e ansiedade (Xavier et al., 2001b), análise de fatores de risco concomitantes para doenças cerebrovasculares e função 
Tabela 2. O procedimento Recordação Seletiva em estudos brasileiros

\begin{tabular}{|c|c|c|c|c|}
\hline Estudo & tarefa & objetivo artigo/uso do SR & participantes & procedimentos/escores \\
\hline Netto et al., 2013 & $\begin{array}{l}\text { TEB (Buschke \& Fuld, } \\
\text { 1974) }\end{array}$ & $\begin{array}{l}\text { avaliar memória episódica viso- } \\
\text { verbal em treinamento de MT }\end{array}$ & $\begin{array}{l}20 \text { idosos ( } 11 \text { experimentais } \\
\text { e } 9 \text { controles) }\end{array}$ & $\begin{array}{l}\text { escores: evocação livre, pistas e } \\
\text { evocação livre total }\end{array}$ \\
\hline Bolshaw et al., 2011 & $\begin{array}{l}\text { TEB (Buschke \& Fuld, } \\
\text { 1974; Petersen et al., } \\
\text { 1992, 1994) }\end{array}$ & $\begin{array}{l}\text { comparar pacientes com } \\
\text { transtorno do pânico e saudáveis }\end{array}$ & 15 pânico 15 controles & $\begin{array}{l}\text { aprendizagem verbal e memória } \\
\text { episódica viso-espacial } \\
\text { (armazenamento, retenção e } \\
\text { evocação de memória de longo } \\
\text { prazo) }\end{array}$ \\
\hline $\begin{array}{l}\text { Brooks, Borela, \& } \\
\text { Fragoso, } 2011\end{array}$ & $\begin{array}{l}\text { SRT (subteste da } \\
\text { BRB-N) }\end{array}$ & $\begin{array}{l}\text { avaliar cognitivamente pacientes } \\
\text { com esclerose múltipla }\end{array}$ & $\begin{array}{l}39 \text { esclerose múltipla } 47 \\
\text { controles }\end{array}$ & não informado \\
\hline Trentini et al., 2009 & $\begin{array}{l}\text { TESLP (Buschke \& } \\
\text { Fuld, 1974; Petersen et } \\
\text { al., 1992, 1994) }\end{array}$ & $\begin{array}{l}\text { avaliar as habilidades cognitivas } \\
\text { de idosos viúvos }\end{array}$ & $\begin{array}{l}30 \text { viúvos } 30 \text { controles } \\
\text { casados }\end{array}$ & evocação livre + pistas \\
\hline $\begin{array}{l}\text { Bicca \& Argimon, } \\
2008\end{array}$ & TESLP (Buschke)+ & $\begin{array}{l}\text { comparar cognitivamente idosas } \\
\text { usuárias e não usuárias de } \\
\text { benzodiazepínicos }\end{array}$ & $\begin{array}{l}124 \text { idosas } \\
\text { institucionalizadas }\end{array}$ & evocação livre + pistas total \\
\hline Bottino et al., 2008 & SRT & $\begin{array}{l}\text { traçar perfil cognitivo de } \\
\text { pacientes em uma clínica de } \\
\text { memória }\end{array}$ & 104 pacientes & não informado \\
\hline Almeida et al., 2007 & BSRP (Buschke, 1973) & $\begin{array}{l}\text { avaliar alterações cognitivas em } \\
\text { pacientes com hipotireoidismo } \\
\text { subclínico (HS) }\end{array}$ & $\begin{array}{l}65 \text { pacientes com HS e } 31 \\
\text { eutireoidianos }\end{array}$ & $\begin{array}{l}\text { lista de } 12 \text { palavras através } \\
\text { de } 12 \text { ensaios. Palavras não } \\
\text { lembradas eram repetidas. } \\
\text { Escores: armazenamento, } \\
\text { recuperação, RCLD, RLDR, } \\
\text { intrusões, evocação tardia (20') } \\
\text { e reconhecimento }\end{array}$ \\
\hline Maineri et al., 2007 & $\begin{array}{l}\text { TESLP (Buschke; } \\
\text { Petersen et al., 1992) }\end{array}$ & $\begin{array}{l}\text { análise de fator de risco } \\
\text { concomitante para doença } \\
\text { cerebrovascular e função } \\
\text { cognitiva }\end{array}$ & 46 participante & $\begin{array}{l}\text { evocação livre + pistas, livre e } \\
\text { tardio }\end{array}$ \\
\hline Zachi et al., 2007 & $\begin{array}{l}\text { BSRT (Spreen \& } \\
\text { Strauss, 1998) }\end{array}$ & $\begin{array}{l}\text { avaliar alterações cognitivas } \\
\text { de pacientes com histórico } \\
\text { de intoxicação por vapor de } \\
\text { mercúrio }\end{array}$ & $\begin{array}{l}26 \text { expostos ao mercúrio } 20 \\
\text { controle }\end{array}$ & $\begin{array}{l}\text { número total de palavras, } \\
\text { recuperação de longa duração, } \\
\text { armazenamento de longa } \\
\text { duração, RCLD, RLDR, } \\
\text { recordação tardia, recordação } \\
\text { com pistas, intrusões }\end{array}$ \\
\hline Xavier et al., 2006 & $\begin{array}{l}\text { TEB (Buschke, 1974; } \\
\text { Petersen et al., 1992) }\end{array}$ & $\begin{array}{l}\text { avaliar desempenho de idosos } \\
\text { saudáveis com baixa escolaridade } \\
\text { em testes neuropsicológicos }\end{array}$ & 200 idosos & $\begin{array}{l}\text { evocação livre }+ \text { pistas total } \\
\text { número total }\end{array}$ \\
\hline $\begin{array}{l}\text { Argimon \& Stein, } \\
2005\end{array}$ & $\begin{array}{l}\text { TBLLP (Buschke \& } \\
\text { Fuldt, 1974)+ }\end{array}$ & $\begin{array}{l}\text { avaliar mudanças cognitivas } \\
\text { em indivíduos muito idosos no } \\
\text { período de três anos }\end{array}$ & 66 idosos (início) 46 (final) & evocação livre + pistas total \\
\hline $\begin{array}{l}\text { Argimon, Stein, } \\
\text { Xavier, \& Trentini, } \\
2004\end{array}$ & $\begin{array}{l}\text { TESLPB (Buschke e } \\
\text { Fuld, 1974)+ }\end{array}$ & $\begin{array}{l}\text { avaliar a cognição de idosos na } \\
\text { diversidade de atividades de lazer }\end{array}$ & 121 idosos & não informado \\
\hline Cunha et al., 2004 & $\begin{array}{l}\text { BSRT (Spreen \& } \\
\text { Strauss, 1998) }\end{array}$ & $\begin{array}{l}\text { avaliar desempenho cognitivo em } \\
\text { dependentes de cocaína e crack }\end{array}$ & $\begin{array}{l}15 \text { dependentes de cocaína/ } \\
\text { crack } 15 \text { controles }\end{array}$ & $\begin{array}{l}\text { escores: recuperação total, } \\
\text { recuperação de longa duração, } \\
\text { recuperação de curta duração, } \\
\text { armazenamento de longa } \\
\text { duração, RCLD, RLDR, } \\
\text { pistas, recuperação após } 15 \text { ', } \\
\text { reconhecimento }\end{array}$ \\
\hline Bottino et al., 2002 & SRT & $\begin{array}{l}\text { avaliar tratamento com } \\
\text { inibidor da acetilcolinesterase } \\
\text { e treinamento cognitivo em } \\
\text { pacientes com DA leve }\end{array}$ & 6 pacientes com DA leve & não informado \\
\hline $\begin{array}{l}\text { Brito, Araujo \& Papi, } \\
2002\end{array}$ & BSRT & estudo de caso & mulher 49 anos & não informado \\
\hline $\begin{array}{l}\text { Carvalho \& Assencio- } \\
\text { Ferreira, } 2002\end{array}$ & $\begin{array}{l}\text { SRT/TMVS (Buscke } \\
\text { fuld, 1974) }\end{array}$ & $\begin{array}{l}\text { avaliação funcional em } \\
\text { habilidades fonológicas no } \\
\text { envelhecimento normal e na DA }\end{array}$ & $8 \mathrm{DA}$ e 10 normais & não informado \\
\hline
\end{tabular}


Tabela 2. O procedimento Recordação Seletiva em estudos brasileiros (cont.)

\begin{tabular}{lllll}
\hline \multicolumn{1}{c}{ Estudo } & \multicolumn{1}{c}{ tarefa } & \multicolumn{1}{c}{ objetivo artigo/uso do SR } & \multicolumn{1}{c}{ participantes } & \multicolumn{1}{c}{ procedimentos/escores } \\
\hline Xavier et al., 2002 & FCSR & $\begin{array}{l}\text { avaliar desempenho cognitivo } \\
\text { no luto }\end{array}$ & 77 idosos & evocação total (livre+pistas) \\
Degenszajn et al., 2001 & $\begin{array}{l}\text { FCSR (Peterson et al., } \\
\text { avaliar distúrbio da codificação } \\
\text { em problemas de aprendizado e } \\
\text { memória e investigar a taxa de } \\
\text { esquecimento na DA }\end{array}$ & $\begin{array}{l}\text { DA leve e moderada15 } \\
\text { controles }\end{array}$ & $\begin{array}{l}\text { evocação livre e livre+ pistas em } \\
\text { cada ensaio, em 30' e 24hs }\end{array}$ \\
Comparar cognição de idosos \\
Xavier et al., 2001
\end{tabular}

Nota: mais estudos que utilizaram a versão de Petersen et al. (1992) e citaram outros estudos. BSR - Buschke Selective Reminding; BSRP - Buschke's Selective Reminding Procedure (1973); BSRT - Buschke's Selective Reminding Test; DA - doença de Alzheimer; HS (hipotireoidismo subclinico); MT - memória de trabalho; RBRBNT - Rao's Brief Repeatable Battery of Neuropsychological Tests; RCLD recuperação consistente de longa duração; RLD recuperação de longa duração randômica; SRT - Selective Reminding Test; SR-V - Selective Reminding - Verbal; TBLLP - Teste de Buschke Lembranças Livres e com Pista; TEB - Teste de Evocação de Buschke; TESLP - Teste de Evocação Seletiva Livre e com Pistas; TESLPB - Teste de Evocação Seletiva Livre e com Pistas de Buschke; TMVS - Teste de Memória Verbal Seletiva; TRB - Teste de Recuperação de Buschke.

cognitiva (Maineri, Xavier, Berleze, \& Moriguchi, 2007), comparações do desempenho cognitivo de idosos que utilizam benzodiazepínicos a controles (Bicca \& Argimon, 2008), relações entre variáveis demográficas, como viuvez, luto e cognição (Trentini, Werlang, Xavier, \& Argimon, 2003; Xavier, Ferraz, Trentini, Fretias, \& Moriguchi, 2002), investigação da memória em indivíduos saudáveis de baixa escolaridade (Xavier et al., 2006), avaliação das diferenças entre pacientes com DA e grupo controle em relação à codificação no desempenho de aprendizagem e memória tardia (Degenszajn, Caramelli, Caixeta, \& Nitrini, 2001), entre pacientes com transtorno do pânico e saudáveis (Bolshaw et al., 2011), entre idosos com transtorno depressivo maior e controles (Xavier et al., 2001a) e avaliação cognitiva de idosos que realizam diferentes atividades físicas (Argimon et al., 2004). Ainda, alguns estudos não apresentaram de forma clara o procedimento de recordação seletiva adotado ou os resultados encontrados através dele (Bottino et al., 2002; Bottino et al., 2008; Brito, Araújo \& Papi, 2002; Carvalho \& Assenciona-Ferreira, 2002).

Dentre os estudos nacionais recuperados em revisão, não foram encontrados quaisquer que tivessem o objetivo de construir, adaptar ou verificar as propriedades psicométricas de instrumentos que utilizam o procedimento de recordação seletiva. Em alguns estudos, a falta dos procedimentos de validação impede que interpretações mais robustas sejam derivadas. Por exemplo, Xavier et al. (2006), ao compararem seus resultados com os de outros países, apontaram dificuldades de generalizações, pois a falta de instrumentos adaptados poderia ter gerado o desempenho inferior das amostras brasileiras em relação ao observado em amostras de outros países. Sendo assim, a carência de um instrumento que utilize o procedimento de recordação seletiva constitui-se em uma lacuna na produção científica nacional, apresentando um prejuízo para o contexto clínico de avaliação da memória de idosos. Além disso, a maior parte dos estudos brasileiros analisados (Tabela 2) fez uso somente de alguns escores do método de recordação seletiva, o que, por vezes, dificulta a comparação entre resultados. Ainda, vale ressaltar que, dos 20 artigos brasileiros analisados que utilizaram o procedimento de recordação seletiva, seis não descreveram no corpo do artigo, informações quanto aos procedimentos de aplicação do teste ou aos escores adotados. Da mesma forma, alguns outros artigos, por vezes, deixaram de apresentar os procedimentos de aplicação, o número de itens e de ensaios ministrados, a descrição dos itens e/ou os escores utilizados.

\section{Considerações Finais}

O presente artigo teve como objetivo apresentar os aspectos teóricos e metodológicos do procedimento de recordação seletiva. Ao comparar o procedimento padrão de recordação da lista e o procedimento de recordação seletiva, constata-se que a maior diferença entre eles está relacionada à não apresentação de itens já evocados em ensaios imediatamente anteriores. Essa mudança no procedimento estaria relacionada à maior rapidez da aprendizagem (MacLeod, 1985). Ambas as vantagens estão relacionadas ao direcionamento da atenção e do esforço para itens ainda não aprendidos.

O procedimento tradicional tem suas vantagens na medida em que a lista pode ser aprendida de forma completa e padronizada. Por sua vez, o procedimento de recordação seletiva tem como característica uma apresentação personalizada, tendo em vista que os itens reapresentados dependem do desempenho do avaliando. Assim, através deste, é possível monitorar as diferenças individuais na realização da tarefa.

Destaca-se que o procedimento de recordação seletiva possibilita avaliar quais itens foram constantemente recordados (aprendidos). O procedimento tradicional, porém, permite a recordação a cada ensaio em função da constante reapresentação, o que limita a investigação dos erros de aprendizagem cometidos pelo avaliado em cada ensaio. Ou seja, apesar de os itens serem mais consistentemente evocados, esse efeito pode se dar em razão de sua apresentação repetida e não de uma aprendizagem mais duradoura. Além disso, é possível que a reapresentação somente dos itens não recordados facilite a organização subjetiva da lista de forma a auxiliar à aprendizagem (Buschke \& Fuld, 1974). 
Outra vantagem apresentada pelo procedimento de recordação seletiva é a disponibilização de escores relacionados ao que já fora aprendido da lista e o que ainda não fora. $\mathrm{O}$ uso do método tradicional não propicia tais dados, tendo em vista a impossibilidade de saber se o item está sendo evocado de um armazenamento de curta duração ou de longa duração, já que existe a apresentação completa da lista em todos os ensaios.

Em relação ao uso dos métodos, de acordo com a revisão realizada, foi possível constatar que os pesquisadores fazem uso de diferentes tarefas, com formas de aplicação do método distintas. Por exemplo, diferenças no número de itens da tarefa, uso ou não de tarefas distratoras entre os ensaios, utilização de categorias semânticas para codificação dos itens, recordações com ou sem pista, tarefas de reconhecimento agregadas, uso de apenas alguns dos escores nas avaliações, entre outros. Apesar de cada tarefa apresentar algumas vantagens de acordo com o objetivo do estudo, o uso de diferentes procedimentos faz com que as generalizações se tornem mais difíceis (Loring \& Papanicolaou, 1987).

Destaca-se a necessidade de instrumentos adaptados e com normas adequadas à população brasileira, para que a interpretação dos resultados seja mais fidedigna e válida. Estudos longitudinais em amostras brasileiras, reproduzindo os achados de estudos internacionais, poderiam ser de grande valia no auxílio da avaliação das demências, tendo em vista a vasta literatura apontando o potencial de diagnóstico diferencial e preditivo do procedimento. Indica-se a necessidade de estudos futuros, especialmente de tradução e adaptação de tarefas e testes com o uso do procedimento de recordação seletiva ou mesmo o desenvolvimento de instrumentos com essas características, os quais poderiam auxiliar nas avaliações neuropsicológicas de memória e aprendizagem.

\section{Referências}

Abrisqueta-Gomes, J. (2013). Memória e envelhecimento cognitivo saudável. In L. F. Malloy-Diniz, D. Fuentes, \& R. Consenza (Orgs.), Neuropsicologia do Envelhecimento: uma abordagem multidimensional. Porto Alegre: Artmed.

Almeida, C., Vaisman, M., Costa, A. J. L., Reis, F. A. A., Reuters, V., Teixeira, P.,... Brasil, M. A. (2007). Are neuropsychological changes relevant in subclinical hypothyroidism? Arquivos Brasileiros de Endocrinologia, 52(4), 606-611. doi:10.1590/ S0004-27302007000400016

Argimon, I. L., \& Stein, L. M. (2005). Habilidades cognitivas em indivíduos muito idosos: Um estudo longitudinal. Caderno de Saúde Pública, 21(1) 64-72. doi:10.1590/S0102311X2005000100008

Argimon, I. L., Stein, L. M., Xavier, F. M. F., \& Trentini, C. M. (2004). O impacto de atividades de lazer no desenvolvimento cognitivo de idosos. Revista Brasileira de Ciências do Envelhecimento Humano, 1(1), 38-47.

Atkinson, R. C., \& Shiffrin, R. M. (1968) Human memory: A proposed system and its control processes. In K. W. Spence \& J. T. Spence (Eds.), The psychology of learning and motivation: Advances in research and theory. New York. Academic Press.
Battig, W. F. (1965). Procedural problems in paired-associate learning research. Psychonomic Press.

Bell, B. D., Fine J., Dow, C., Seidenberg, M., \& Hermann, B. P. (2005). Temporal lobe epilepsy and the Selective Reminding Test: The conventional 30-minute delay suffices. Psychological Assessment, 17(1), 103-109. doi:10.1037/10403590.17.1.103

Bertolucci, P. H., Okamoto, I. H., Neto, J. T., Ramos, L. R., \& Brucki, S. M. D. (1998). Desempenho da população brasileira na bateria neuropsicológica do Consortium to Establish a Registry for Alzheimer's Disease (CERAD). Revista de psiquiatria clínica, 25(2), 80-83.

Bicca, M. G., \& Argimon, I. I. L. (2008). Habilidades cognitivas e uso de benzodiazepínicos em idosas institucionalizadas. Jornal Brasileiro de Psiquiatria, 57(2), 133-138. doi:10.1037/02786133.24.2.225

Bishop, E. G., Dickson, A. L., \& Allen, M. T. (1990). Psychometric intelligence and performance on selective reminding. The Clinical Neuropsychologist 4, 141-150. doi:10.1080/13854049008401507

Bolshaw, M., Greca, D. V., Nardi, A. E., Cheniaux, E., Fonseca, R. P., \& Fernandez, J. L. (2011). Funções cognitivas no transtorno do pânico: Um estudo comparativo com controles saudáveis. Psico, 42(1), 87-97.

Bottino, C. M. C., Carvalho, I. A. M., Alvarez, A. M. M. A., Avila, R., Zukauskas, P. R., Bustamante, S. E. Z., ... Camargo, C. H. P. (2002) Reabilitação cognitiva em pacientes com Doença de Alzheimer. Arquivos de Neuropsiquiatria, 60(1), 70-79.

Bottino, C. M. C., Zucollo, P., Moreno, M. D. P. Q., Gil, G., Cid, C. G., Campanha, E. V., ... Camargo, C. H. P. (2008). Assessment of memory complainers in São Paulo, Brazil. Dementia \& Neuropsychologia, 2(1), 52-56.

Bower, G. H (2000). A brief history of memory research. In E. Tulving \& F. I. M. Craik (Eds.), The Oxford Handbook of Memory (pp. 3-32). Oxford University Press, New York.

Brito, G. N. O., Araujo, G. R. B., \& Papi, J. A. (2002). Neuropsychological, neuroimage and psychiatric aspects of primary Sjögren's syndrome. Arquivos de neuropsiquiatria, 60(1). 28-31. doi:10.1590/S0004-282X2002000100006

Brooks, J. B. B., Borela, M. C. M., \& Fragoso, Y. D. (2011). Assessment of cognition using the Rao's Brief Repeatable Battery of Neuropsychological Tests on a group of Brazilian patients with multiple sclerosis. Arquivos de Neuropsiquiatria, 69(6), 887-891. doi:10.1590/S0004-282X2011000700007

Brown, S. C., \& Craik, F. I. M. (2000). Encoding and retrieval of information. In E. Tulving \& F. I. M. Craik (Orgs), The Oxford Handbook of Memory (pp 93-107). New York: Oxford University Press.

Bueno, O. F. A., Bertolucci, P. H. F., Oliveira, M. G. M., \& Abrisqueta-Gomez, J. (2008). Effects of semantic relations, repetition of words, and list length in word list recall of Alzheimer's patients. Arquivos de Neuro-Psiquiatria, 66(2b), 312-317. doi:10.1590/S0004-282X2008000300005

Buschke, H. (1973). Selective Reminding for Analysis of Memory and Learning. Journal of Verbal Learning and Verbal Behavior, 12(5), 543-550. doi:10.1016/S0022-5371(73)80034-9

Buschke, H. (1984). Cued recall in Amnesia. Journal of Clinical and Experimental Neuropsychology, 6, 433-440. doi:10.1080/01688638408401233 
Buschke, H., \& Fuld, P. A. (1974). Evaluating storage, retention, and retrieval in disordered memory and learning. Neurology, 24, 1019-1025. doi:10.1212/WNL.24.11.1019

Campo, P., \& Morales, M. (2004). Normative data and reliability for a Spanish version of the verbal Selective Reminding Test. Archives of Clinical Neuropsychology, 19(3), 421-435. doi:10.1016/S0887-6177(03)00075-1

Carvalho, I. A. M., \& Assencio-Ferreira, V. J. (2002). Análise das habilidades fonológicas no envelhecimento normal e na doença de Alzheimer. Revista CEFAC, 4, 235-240.

Crodfelter, C. J., Dickson, A. L., Newton, C. W., \& Johnson, R. B. (1987). Alternate forms of selective reminding for children. The Clinical Neuropsychologist, 1, 243-249. doi:10.1080/13854048708520058

Culang-Reinlieb, M. E., Sneed, J. R., Keilp, J. G., \& Roose, S. P. (2012). Change in cognitive functioning in depressed older adults following treatment with sertraline or nortriptyline. International Journal of Geriatric Psychiatry, 27(8), 777-784. doi:10.1002/gps.2783

Cunha, P. J. Nicastri, S., Gomes, L. P. Moino, R. M., \& Peluso, M. A. (2004) Alterações neuropsicológicas em dependentes de cocaína/crack internados: Dados preliminares. Revista Brasileira de Psiquiatria, 26(2), 103-106.

Deloire, M. S., Bonnet, M. C., Salort, E., Arimone, Y, Boudineau, M. Petry, K. G., \& Brochet, B. (2006). How to detect cognitive dysfunction at early stages of multiple sclerosis? Multiple Sclerosis, 12(4), 445-452. doi:10.1191/1352458506ms1289oa

Degenszajn, J., Caramelli, P., Caixeta, L., \& Nitrini, R. (2001). Encoding process in delayed recall impairment and rate of forgetting in Alzheimer's Disease. Arquivos de Neuropsiquiatria, 59(2-A), 171-174. doi:10.1590/S0004282X2001000200003

Di Stefano, F., Epelbaum, S., Coley, N., Cantet, C., Bakardjian, H., Lista, S., ... Andrieu, S. (2014). Free and Cued Selective Reminding Test (FCSRT) performance in the prediction of Alzheimer's Disease Dementia: Data from a large prevention trial (Guidage). Alzheimer's \& Dementia: The Journal of the Alzheimer's Association, 10(4), 721-271. doi:10.1016/j. jalz.2014.05.1336

Gigi, A., Schnaider-Beeri, M., Davidson, M., \& Prohovnik, I., (1999). Validation of a Hebrew selective reminding test. Israel Journal of Psychiatry and Related Sciences, 36, 11-17.

Glanzer, M., \& Cunitz, A. R. (1966). Two storage mechanisms in free recall. Journal of Verbal Learning and Verbal Behavior, 5, 351-360. doi:10.1016/S0022-5371(66)80044-0

Grober, E., \& Buschke, H. (1987). Genuine memory meficits in mementia. Developmental Neuropsychology, 3(1), 13-16. doi:10.1080/8756564870954036

Grober E., Hall, C. B., Lipton, R. B., Zonderman, A. B., Resnick, S. M., \& Kawas, C. (2008a). Memory impairment, executive dysfunction, and intellectual decline in preclinical Alzheimer's disease. Journal International Neuropsychological Society, 14(2), 266-78. doi:10.1017/S1355617708080302

Grober, E., Hall, C., Sanders, A., \& Lipton, R. B. (2008b). Free and cued selective reminding distinguishes Alzheimer's Disease from vascular dementia. Journal of the American Geriatrics Society, 56(5), 944-946. doi:10.1111/j.15325415.2008.01652.x
Grober, E., Lipton, R. B., Hall, C., \& Crystal, H. (2000). Memory impairment on free and cued selective reminding predicts dementia. Neurology, 54(4), 827-832. doi:10.1212/ WNL.54.4.827

Grober, E., Merling, A., Heimlich, T., \& Lipton, R. B. (1997). Free and cued selective reminding and selective reminding in the elderly. Journal of Clinical and Experimental Neuropsychology, 19(5), 643-654. doi:10.1080/01688639708403750

Grober E., Sanders, A. E., Hall, C. \& Lipton, R. B. (2010). Free and cued selective reminding identifies very mild dementia in primary care. Alzheimer Disease Association Disorders, 24(3), 284-90. doi:10.1097/WAD.0b013e3181cfc78b

Hannay, J. H., \& Levin, H. S. (1985). Selective Reminding Test: An examination of the equivalence of four forms. Journal of Clinical and Experimental and Neuropsychology, 7, 251-263. doi:10.1080/01688638508401258

Hubley, A. M., \& Tombaugh, T. N. (2002). Memory Test for Older Adults (MTOA). Torronto: Multi-Health System.

Krupp, L. B., Christodoulou, C., Melville, P., Scherl, W. F.; Pai, L. Y. Muenz, L. R., ...Wishart, H. (2011). Multicenter randomized clinical trial of donepezil for memory impairment in multiple sclerosis. Neurology, 76(17), 1500-1507. doi:10.1212/ WNL.0b013e318218107a

Izquierdo, I. (2011). Memória (2th ed.). Porto Alegre: Artmed.

Izquierdo, I. A., Myskiw, J. C., Benetti, F., \& Furini, C. R. G. (2013). Memória: Tipos e mecanismos-achados recentes. Revista USP, (98), 9-16. Retrieved from http://www.revistas.usp.br/revusp/ article/view/69221

Karpicke, J. D., \& Roediger, H. L. (2008). The critical importance of retrieval for learning. Science, 319(5865), 966-968. doi:10.1126/science. 1152408

Larrabbe, G. J., Trahan, D. E., Curtiss, G., \& Levin, H. S. (2000). Normative data for a six-trial administration of the Verbal Selective Reminding Test. Neuropsychology, 2, 173-182. doi:10.1076/1385-4046(200002)14:1;1-8;FT110

Lemos, R., Duro, D., Simões, M. R., \& Santana, I. (2014). The free and cued selective reminding test distinguishes frontotemporal dementia from Alzheimer's disease. Archives of Clinical Neuropsychology, 29(7), 670-679. doi:10.1093/ arclin/acu031

Lezak, M. D. (1995). Neuropsychological Assessment (3rd ed.). New York: Oxford University Press.

Loring, D. W., \& Papanicolaou, A. C. (1987). Memory assessment in neuropsychology: theoretical considerations and practical utility. Journal of Clinical and Experimental Neuropsychology, 9, 340-358. doi:10.1080/01688638708405055

MacLeod, C. (1985). Learning a list for free recall: selective reminding versus the standard procedure. Memory \& Cognition, 13(3), 233-240. doi:10.3758/BF03197686

Maineri, N. L., Xavier, F. M. F., Berleze, C. M. C., \& Moriguchi, E. H. (2007). Fatores de risco para doença cerebrovascular e função cognitiva em idosos. Arquivos Brasileiros de Cardiologia, 89(3), 158-162. doi:10.1590/S0066$782 \times 2007001500003$

Malloy-Diniz, L. F., Lasmar, V. A. P., Gazinelli, L. S. R., Fuente, D., \& Salgado, J. V. (2007). The Rey Auditory-Verbal Learning Test: Applicability for the Brazilian elderly population. Revista Brasileira de Psiquiatria, 29(4), 324-329. doi:10.1590/S151644462006005000053 
Markowitsch, H. J. (1995) Which brain regions are critically involved in the retrieval of old episodic memory? Brain Research Reviews, 21(2), 117-127. doi:10.1016/01650173(95)00007-0

McCormick, C., Moscovitch, M., Protzner, A. B., Huber, C. G., \& McAndrews, M. P. (2010). Hippocampalneocortical networks differ during encoding and retrieval of relational memory: Functional and effective connectivity analyses. Neuropsychologia, 48, 3272-3281. doi:10.1016/j. neuropsychologia.2010.07.010

Miller, G. A. (1956). The magical number seven, plus or minus two: Some limits on our capacity for processing information. Psychological Review, 63(2), 81-97. doi:10.1037/h0043158

Morales, M., Campo, P., Fernández, A., Moreno, D., Yáñez, J., \& Sañudo, I. (2010). Normative data for a six-trial administration of a Spanish version of the Verbal Selective Reminding Test. Archives of Clinical Neuropsychology, 25(8), 745-761. doi:10.1093/arclin/acq076

Morgan, S. F. (1982). Measuring long-term memory, storage and retrieval in children. Journal of Clinical Neuropsychology, 4, 77-85. doi:10.1080/01688638208401118

Murdock, B. B. (1962). The serial Position Effect of Free Recall. Journal of Experimental Psychology, 64(5) 482-488. doi: $10.1037 / \mathrm{h} 0045106$

Netto, T. M., Greca, D. V., Zimmermann, N., Oliveira, C. R., Teixeira-Leite, H., ... Landeira-Fernandez, J. (2013). Efeito de um programa de treinamento da memória de trabalho em adultos idosos. Psicologia: Reflexão e Crítica, 26(1), 122-135.

Payne, D. G., \& Roediger III, H. L. (1987). Hypermnesia occurs in recall but not in recognition. The American Journal of Psychology, 100(2) 145-165. doi:10.2307/1422400

Petersen, R. C., Smith, G., Kokmen, E., Ivnik, R. J., \& Talangos, E. G. (1992). Memory function in normal aging. Neurology, 42, 396-401. doi:10.1212/WNL.42.2.396

Pillai, J. A., Hall, C. B., Dickson, D. W., Buschke, H., Lipton, R. B., \& Verghese, J. (2011). Association of crossword puzzle participation with memory decline in persons who develop dementia. Journal of International Neuropsycholy Society, 17(6), 1006-1013. doi:10.1017/S1355617711001111

Pyc, M. A., \& Rawson, K. A. (2011). Costs and benefits of dropout schedules of test-restudy practice: Implications for student learning. Applied Cognitive Psychology, 25(1), 87-95. doi:10.1002/acp.1646

Quevedo, J., Martins, M. R., \& Izquierdo, I. (2006). Alterações cerebrais e memória. In Bottino, C. M. C., Laks, J. \& Blay, S. L. (Orgs), Demência e transtornos cognitivos em idosos (pp. 3-12). Rio de Janeiro: Guanabara Koogan.

Remigio, L. F. C. (2010). Hypermnesic recall across repeated tests using mental images and socratic riddles. Enseñanza e Investigación en Psicología, 15(2), 403-416.

Ribeiro, P. C. C., Oliveira, B. H. D., Cupertino, A. P. F. B., Neri, A. L., \& Yassuda, M. S. (2010). Desempenho de idosos na Bateria Cognitiva CERAD: Relações com variáveis sociodemográficas e saúde percebida. Psicologia Reflexão e Crítica, 23(1), 102109.
Solanto, M. V., Gilbert, S. N., Raj, A., Zhu, J., Pope-Boyd, S, Stepak, B., ... Newcorn, J. H. (2007). Neurocognitive functioning in $\mathrm{AD} / \mathrm{HD}$, predominantly inattentive and combined subtypes. Journal of Abnormal Children Psychology, 35(5), 729-744. doi:10.1007/s10802-007-9170-z

Strauss, E., Sherman, E. M. S., \& Spreen, O. (2006). A compendium of neuropsychological tests: Administration, norms, and commentary (3rd ed.). Oxford University Press, New York.

Tombaugh, T. N., \& Schmidt, J. P. (1992). The learning and memory battery (LAMB): Development and standardization. Psychological Assessment, 4, 193-206. doi:10.1037/10403590.4.2.193

Trentini, C. M., Werlang, B. S. G. W., Xavier, F. M. F., \& Argimon, I. I. L. (2003). A relação entre variáveis de saúde mental e cognição em idosos viúvos. Psicologia: Reflexão e Crítica, 22(2), 236-243.

Tulving, E. (1962). Subjective organization in Free Recall of "unrelated" words. Psychological Review, 69(4), 344-354. doi: $10.1037 / \mathrm{h} 0043150$

Tulving, E., \& Craik, F. I. M. (2000). The handbook of memory. New York: Oxford University Press.

Tulving, E., \& Pearlstone, Z. (1966). Avaliability versus Acessibility of information in memory for words. Journal of Verbal Learning and Verbal Behavior, 5, 381-391. doi:10.1016/ S0022-5371(66)80048-8

Xavier, F. M. F., Argimon, I., Zuppo, L. P., Lucchesi, L., Helyany, C., C. V., \& Trentini, C. M. (2006). O desempenho em testes neuropsicológicos de octagenários não-dementes e com baixa escolaridade em duas comunidades do sul do Brasil. Psico (PUCRS), 37, 221-231.

Xavier, F. M. F., Ferraz, M. P. T, Bertollucci, P., Poyares, D., \& Moriguchi, E. H. (2001a). Episódio depressivo maior, prevalência e impacto sobre qualidade de vida, sono e cognição em octogenários. Revista Brasileira de Psiquiatria, 23(2), 62-70.

Xavier, F. M., Ferraz, M. P., Trentini, C. M., Argimon, I., Bertolucci, P. H., Payares, D., \& Morigughi, E. H. (2001b). Generalized anxiety disorder in a population aged 80 years and older. Revista de Saúde Pública, 35(3), 294-302. doi:10.1590/S003489102001000300013

Xavier, F. M., Ferraz, M. P., Trentini, C. M., Freitas, N. K., \& Moriguchi, E. H. (2002). Bereavement-related cognitive impairment in an oldest-old community-dwelling Brazilian sample. Journal of Clinical and Experimental Neuropsychology, 24(3), 294-301). doi:10.1076/jcen.24.3.294.983

Zachi, E. G., Ventura, D. F., Faria, M. A. M., \& Taub, A. (2007). Neuropsychological dysfunction related to earlier occupational exposure to mercury vapor. Brazilian Journal of Medical and Biological Research, 40(3), 425-433. doi:10.1590/S0100879X2007000300019

Wenger, M. J., Negash, S., Petersen, R. C., \& Petersen, L. (2010). Modeling and estimating recall processing capacity: Sensitivity and diagnostic utility in application to mild cognitive impairment. Journal of Mathematical Psychology, 54(1), 7389. doi:10.1016/j.jmp.2009.04.012

Recebido em 04.07.2014

Primeira decisão editorial em 16.09.2015

Versão final em 15.11.2015

Aceito em 01.02.2016 